\title{
Predictors of adverse drug reactions in geriatric patients: An exploratory study among cancer patients
}

\author{
S. George Sneha, Kalpana Simhadri, Viswam K. Subeesh, S.Varghese Sneha
}

\begin{abstract}
Objectives: The objective of this study was to study the predictors of adverse drug reactions (ADRs) among geriatric patients in the Department of Medical Oncology. Methods: A hospital-based prospective observational study was carried out among 153 inpatients in the Department of Medical Oncology for 6 months. Patients above 60 years of age with a confirmed history of malignancy were included in the study. The potential risk factors for ADR were defined in relation to the patient and chemotherapeutic regimen and relationship between them was assessed by univariate and multivariate logistic regression analysis. Results: Among 153 patients, 94 (64.43\%) experienced ADRs. The mean ADR per patient was 0.88 \pm I.2. The common ADRs found were alopecia $(30.18 \%)$ and diarrhea $(28.68 \%)$. Risk estimates revealed that there was a significant association between smokers (odds ratio [OR] = I0.326; $95 \%$ confidence interval $[\mathrm{Cl}] 2.345-45.47, P=0.00 \mathrm{I})$, alcoholics $(\mathrm{OR}=10.897 ; 95 \% \mathrm{Cl} 2.479-47.902, P=0.00 \mathrm{I})$, increasing age $(\mathrm{OR}=2.22$; $95 \% \mathrm{Cl}$ $\mathrm{I} .698-2.909, P=0.00 \mathrm{I})$, overweight $(\mathrm{OR}=16.68 ; 95 \% \mathrm{Cl} 2.179-\mathrm{I} 27.74 \mathrm{I}, P=0.00 \mathrm{I})$, and male participants $(\mathrm{OR}=0 . \mathrm{I} 43 ; 95 \% \mathrm{Cl} 0.05-0.390 \mathrm{P}=0.00 \mathrm{I})$ with the development of ADRs. The risk of carboplatin $(\mathrm{OR}=\mathrm{I} 3.359 ; 95 \% \mathrm{Cl} 3.056-58.406 \mathrm{P}=0.00 \mathrm{I})$ and 5-fluorouracil $(\mathrm{OR}=\mathrm{I} .93895 \% \mathrm{Cl} \mathrm{I} .266-2.935$ $P=0.00 \mathrm{I})$ use and occurrence of ADRs were also found to be high. Conclusion: The study findings showed that smoking, alcohol consumption, age more than 70 years, and overweight had a high risk for developing ADRs in geriatric patients who underwent chemotherapy. The independent risk factors identified should be targeted for preventive measures to improve anticancer agent prescription and reduce the risk of ADRs.
\end{abstract}

Key words: Adverse drug reactions, cancer, chemotherapy, geriatrics, predictors

\section{Introduction}

Cancer is one of the heterogeneous diseases that is characterized by uncontrolled cellular growth, local tissue invasion, and distant metastases. ${ }^{[1]}$ The increased life expectancy is associated with increasing numbers of individuals, and hence, different diseases become more prevalent with the increase in age. Aging is a high-risk factor for cancer, and approximately $60 \%$ of all cancers and $70 \%$ of cancer mortality occur in persons aged 65 years and over. ${ }^{[2]}$ Adverse drug reactions (ADRs) is most commonly observed in the elderly patients. Accurate risk prediction tool has not yet been sufficiently used in clinical practice. ${ }^{[3]}$ The prevalence of ADRs increases with age and twice as much in patients aged 65 and above. Polypharmacy, gender, and genetic predisposition are the factors that are used to determine the occurrence of ADRs in this population.

An important aspect to consider in the elderly age group is that they have different needs compared to their younger counterparts. Pharmacotherapy of the elderly is complex because of age-related physiologic changes, the presence of multiple comorbidities, and the use of multiple medications. The increased susceptibility of ADRs is due to changes in drug pharmacokinetic and pharmacodynamic parameters. ${ }^{[4,5]}$ These patients may be less able to tolerate certain cancer treatments, and thus, this leads to extremely challenging prescribing scenarios as they have variable pharmacokinetic and pharmacodynamic profiles along with other comorbidities and the presence of current polypharmacy even before the inclusion of any additional cancer therapy. ${ }^{[6]}$

The treatment for cancer includes surgery, radiotherapy, and/or systemic medications (chemotherapy, endocrine therapy, and/or biologic therapy with targeted agents). Several classes of chemotherapeutic agents are used in cancer treatment,

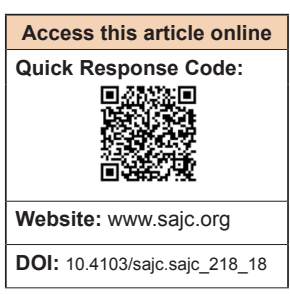

for example, alkylating agents, antimetabolites, monoclonal antibodies, platinum-containing compounds, and hormonal drugs. The choice of treatment modalities regarding radiotherapy, chemotherapy, or even surgery becomes a difficult decision for the doctor, patients, and their relatives. More often elderly patients are treated with conservative or less aggressive treatment. Many of the prospective clinical trials conducted have excluded this group of patients from the trials, and thus no worthwhile clinical studies were accommodating for this age group especially in an Indian setup. Therefore, there seems to be an imminent need to integrate and optimize treatment planning for elderly cancer patients to achieve an improved tumor control, quality of life, and survival with better healthcare. ${ }^{[7]}$

Chemotherapy has been a mainstay of treatment for cancer patients, and older adults are more vulnerable to chemotherapy toxicity or ADRs. ${ }^{[8]}$ Aging is a diverse process which shows characteristic changes in physiology and organ function and can have an impact on the pharmacology of anticancer therapy. This burden of cancer in the elderly has paved the way for modern approach toward the management of these patients. The study focuses on understanding the prescribing pattern in the field of oncogeriatrics and assess for any predictability to ADRs to improve the care given to this population. ${ }^{[9,10]}$ The increasing number of elderly inpatients demands a detailed investigation of the factors that contribute to their mortality. ${ }^{[1]}$

\section{Methods}

A hospital-based prospective observational study was carried out among 153 cancer patients above the age of 60 years in the Department of Medical Oncology for 6 months. The study excluded patients with psychiatric illness and who did not give consent to participate in the study. The protocol was approved

This is an open access journal, and articles are distributed under the terms of the Creative Commons Attribution-NonCommercial-ShareAlike 4.0 License, whichallows others to remix tweak, and build upon the work non-commercially, as long as appropriate credit is given and the new creations are licensed under the identical terms.

M. S. Ramaiah University of Applied Sciences, Bengaluru, Karnataka, India Correspondence to: Viswam K. Subeesh, E-mail: subeeshkviswam@gmail.com
For reprints contact: reprints@ medknow.com

How to cite this article: Sneha SG, Simhadri K, Subeesh VK, Sneha SV. Predictors of adverse drug reactions in geriatric patients: An exploratory study among cancer patients. South Asian J Cancer 2019;8:130-3. 
by the M. S. Ramaiah Medical College and Hospitals, Ethics Committee and the study abided completely to the guidelines provided by them.

A study conducted by Anindya Sarkar and UP Shahi had observed that the prevalence of ADRs in geriatric patients was found to be 57\%. Expecting similar results in the present study with $95 \%$ confidence interval $(\mathrm{CI})$ and $10 \%$ relative precision, the sample size was worked out to be 85 participants, and it was decided to include around 150 participants. ${ }^{[7]}$ Data were collected from medication charts, case sheets, patients' previous medical records, prescriptions, laboratory report, and by conducting a medication history interview.

The individual cases were analyzed for the prescribing pattern of antineoplastic agents, the independent variables were assessed, and the statistically significant risk factors were considered to be the predictors of ADRs.

All the information, which were collected from patients' case notes, were entered in IBM SPSS (Statistical Package for Social Sciences) statistics for windows, version XX.0 version (International Business Machines Corporation (IBM) Corp., Armonk, N.Y.,USA) for easy accessibility, storage, retrieval, and analysis of the collected data. The relationship between risk factors and ADRs was assessed using univariate and multivariate logistic regression analyses. The risk factors considered for the analysis were increasing age, alcoholics, smokers, obesity, and gender. $P \leq 0.05$ was considered as statistically significant. The risk factors, which were statistically significant, were considered as predictors in the study.

\section{Results}

A total of 153 patients were analyzed of which, 42 (27.45\%) were male, and $111(72.55 \%)$ were female. According to the findings, the majority of the patients belonged to the 60-69 years age group. The median age of the study population was found to be 63 years. The gender variation in each category showed female preponderance in the 60-69 years age group. The morbidity pattern [Table 1] of cancer diseases in the study showed that breast cancer is the most common $(41,26.8 \%)$ followed by esophageal $(26,16.99 \%)$, cervical $(21,13.73 \%)$, stomach $(13,8.5 \%)$, and rectal cancers $(10,6.54 \%)$. The pattern of drugs prescribed [Table 2] in the study revealed that antineoplastics $(292,44 \%)$ were the highest followed by antiemetics $(108,16 \%)$, anti-inflammatory agents $(94,14 \%)$, H2 receptor blockers $(70,11 \%)$, antidiabetics $(20,3 \%)$, and antihypertensives (20,3\%). The drug utilization pattern [Table 3] in the present study showed that platinum-containing compounds $(62,21.23 \%)$ was the most commonly prescribed antineoplastic agents followed by antimetabolites $(39,13.36 \%)$, taxanes $(31,10.62 \%)$, and alkylating agents $(24,8.22 \%)$. The prescribing pattern of antineoplastics [Table 4] was found to be important as they accounted for the majority of ADRs. 5-Fluoro Uracil (5 FU) $(41,21 \%)$ was the most commonly prescribed antineoplastic agent followed by carboplatin (32, 16\%), cyclophosphamide (30,15\%), cisplatin (27, 14\%), and adriamycin $(23,12 \%)$. In majority of the prescriptions, the frequency of antineoplastic agents [Table 5] was found to be three $(44.4 \%)$ followed by two $(30.7 \%)$ and four (17.6\%) drugs per prescription.

The percentage of drugs prescribed by generic name was found South Asian Journal of Cancer Volume 8 - Issue 2 April-June 2019
Table 1: The morbidity pattern $(n=153)$ of cancer among the population

\begin{tabular}{lc}
\hline \multicolumn{2}{c}{ Morbidity pattern of cancer } \\
\hline Disease & $n(\%)$ \\
\hline Breast & $41(27)$ \\
Esophagus & $26(17)$ \\
Cervix & $21(14)$ \\
Stomach & $13(8)$ \\
Rectum & $10(7)$ \\
Lung & $10(7)$ \\
Ovary & $7(5)$ \\
Others & $25(16)$ \\
\hline
\end{tabular}

Others: Bladder, colon, nasopharynx, oropharynx, pancreas, tonsils

Table 2: The pattern of drugs prescribed $(n=666)$ among the population

\begin{tabular}{lc}
\hline \multicolumn{2}{c}{ Pattern of drugs prescribed } \\
\hline Drugs & $n(\%)$ \\
\hline Antineoplastics & $292(44)$ \\
Antiemetics & $108(16)$ \\
Anti-inflammatory & $94(14)$ \\
H2-receptor blockers & $70(11)$ \\
Antihistamines & $29(4)$ \\
Antidiabetic & $20(3)$ \\
Antihypertensives & $20(3)$ \\
Antacid & $9(1)$ \\
Analgesics & $5(1)$ \\
Antiplatelet & $5(1)$ \\
Dyslipidemic & $5(1)$ \\
Others & $9(1)$ \\
\hline
\end{tabular}

Others: Electrolyte replenisher, thyroid hormone supplement, vitamin supplement

Table 3: The categories of antineoplastic agents $(n=292)$ prescribed among the population

\begin{tabular}{lc}
\hline \multicolumn{1}{c}{ Categories of antineoplastic agents prescribed } \\
\hline Category & $n(\%)$ \\
\hline Glucocorticoids & $92(32)$ \\
Platinum-containing compounds & $62(21)$ \\
Antimetabolites & $39(13)$ \\
Taxanes & $31(11)$ \\
Alkylating agents & $24(8)$ \\
Antibiotics & $23(8)$ \\
Monoclonal antibodies & $9(3)$ \\
Epipodophyllotoxins & $5(2)$ \\
Others & $7(2)$ \\
\hline
\end{tabular}

Others: Proteasome inhibitors, camptothecin analogs, vinca alkaloids

Table 4: The prescribing pattern of antineoplastic agents $(n=199)$ among the given population

\begin{tabular}{lc}
\hline \multicolumn{2}{c}{ Prescribing pattern of antineoplastics } \\
\hline Drug & $\boldsymbol{n}(\%)$ \\
\hline 5 fluorouracil & $41(21)$ \\
Carboplatin & $32(16)$ \\
Cyclophosphamide & $30(15)$ \\
Cisplatin & $27(14)$ \\
Adriamycin & $23(12)$ \\
Oxaliplatin & $10(5)$ \\
Paclitaxel & $10(5)$ \\
Others & $26(13)$ \\
\hline
\end{tabular}

Others: Bendamustine, bleomycin, bortezomib, epirubicin, etoposide, tamoxifen, vincristine

to be $87.38 \%$. The study findings revealed that $64.43 \%$ of participants experienced ADRs with antineoplastic agents. The mean ADR per patient was $0.88 \pm 1.2$. The common ADRs were 
found to be alopecia $(41,30 \%)$, diarrhea (39, 29\%), vomiting $(20,15 \%)$, neutropenia $(10,7 \%)$, myelosuppression $(5,3 \%)$, and anemia $(4,2 \%)$. The most common systems affected by ADRs were found to be gastrointestinal system (45\%) followed by the dermatologic system (30\%) and hematologic system (18\%). The severity of the reported reactions was assessed using modified Hartwig Siegel, and accordingly, $47.8 \%$ of the ADRs were graded as moderate and $52.2 \%$ as mild. Since most of the reactions were graded as mild-to-moderate severity, it did not warrant any stoppage or change of drug. Risk estimates [Table 6] revealed that there was a significant association between smokers (odds ratio $[\mathrm{OR}]=10.326 ; 95 \%$ CI 2.345-45.47, $P=0.001)$, alcoholics $(\mathrm{OR}=10.897 ; 95 \%$ CI 2.479-47.902, $P=0.001)$, increasing age $(\mathrm{OR}=2.22 ; 95 \%$ CI 1.698-2.909, $P=0.001)$, overweight $(\mathrm{OR}=16.68 ; 95 \%$ CI 2.179-127.741, $P=0.001)$, and male participants (OR $=0.143 ; 95 \% \mathrm{CI}$ $0.05-0.390 P=0.001)$ and development of ADRs. However, comorbidities failed to show a statistically significant difference in risk of developing ADR. The risk of carboplatin and 5 FU use with the occurrence of ADRs were also found to be high.

\section{Discussion}

This study was conducted to evaluate the predictors of ADRs and to analyze the prescribing pattern of anticancer drugs for geriatric patients in the Department of Medical Oncology. The geriatric population is more prone to the adverse effects of medication than their younger counterparts. Identification and reporting of predictors of ADRs in the elderly are crucial to develop preventive strategies and improve care to the patients. Medical practitioners are oblivious to the factors that lead to adverse events. However, to overcome this, several predictors of ADRs have been identified. ${ }^{[12]}$ In the current study, 153 patients were analyzed, of which, females were found to be higher than the males which are in concordance with the study conducted by Sharma et al., ${ }^{[13]}$ on the pattern of ADRs due to cancer chemotherapy (2015). The predominance of females can be correlated with the morbidity pattern which showed that breast cancer was more prevalent in our study population.

Table 5: The number of antineoplastic agents $(n=153)$ per prescription among the population

\begin{tabular}{lc}
\hline & Number of antineoplastic drugs per prescription \\
\hline Number of drugs prescribed & Number of prescriptions (\%) \\
\hline One & $10(7)$ \\
Two & $47(31)$ \\
Three & $68(44)$ \\
Four & $27(18)$ \\
Five & $1(1)$ \\
\hline
\end{tabular}

Table 6: The risk estimates between predictors and adverse drug reactions

\begin{tabular}{lccc}
\hline \multicolumn{4}{c}{ Risk estimates } \\
\hline Predictors & OR & Upper limit - lower limit & $P$ \\
\hline Smokers & 10.326 & $2.345-45.47$ & 0.001 \\
Alcoholics & 10.896 & $2.479-47.902$ & 0.001 \\
Increasing age & 2.22 & $1.698-2.909$ & 0.001 \\
Overweight & 16.68 & $2.179-127.741$ & 0.001 \\
Male participants & 0.143 & $0.05-0.390$ & 0.001 \\
Carboplatin & 13.359 & $3.056-58.406$ & 0.001 \\
5FU & 1.938 & $1.266-2.935$ & 0.001 \\
\hline
\end{tabular}

$\mathrm{OR}=$ Odds ratio
Majority of the patients in the study belonged to 60-69 years age group which can be correlated with the reason of high morbidity in this particular age group because life expectancy decreases with cancer. ${ }^{[14]}$ The morbidity pattern of cancer diseases in the study showed that breast cancer is the most common (26.8\%) followed by esophageal (16.99\%), cervical $(13.73 \%)$, stomach $(8.5 \%)$, and rectal cancers $(6.54 \%)$. These findings were in accordance with the prospective observational study of chemotherapy-induced ADRs in oncology patients conducted by Chopra et al., ${ }^{[15]}$ in the year 2016. The gender variation in the present study (more number of females) justifies the increasing prevalence of breast cancer.

The drug utilization pattern of chemotherapy in the present study showed that platinum-containing compounds $(21.23 \%)$ was the most commonly prescribed antineoplastic agents followed by antimetabolites (13.36\%), taxanes (10.62\%), and alkylating agents $(8.22 \%)$. In the present study, more than half of the patients received an adjunctive therapy with dexamethasone which was similar to many other studies. The role of dexamethasone in the treatment of cancer is mainly due to the reduction in inflammation, immune response, relief of sickness during chemotherapy, and to improve appetite. All of these findings are in concordance with the study conducted by Saini et al., ${ }^{[16]}$ on the pattern of antineoplastic agents used in the oncology department.

The prescribing pattern of antineoplastics was found to be important as they accounted for the majority of ADRs. 5 FU $(21 \%)$ was the most commonly prescribed antineoplastic agent followed by carboplatin (16\%), cyclophosphamide (15\%), cisplatin (14\%), and adriamycin (12\%). In majority of the prescriptions, the frequency of antineoplastic agents was found to be three $(44.4 \%)$ followed by two $(30.7 \%)$ and four $(17.6 \%)$ drugs per prescription. These findings suggest an increased incidence of polypharmacy in this age group which is also supported by the study conducted by Sharma et al. ${ }^{[13]}$ The percentage of drugs prescribed by generic name was found to be $87.38 \%$. In this study, 101 patients' prescriptions consisted of polytherapy in comparison to monotherapy. However, these findings were contrary to the study conducted by Saini et al. ${ }^{[16]}$ The majority of ADRs in the current study was preventable, highlighting the need for close monitoring and review of patients with body mass index, and multiple comorbidities. Risk estimates revealed that there was a significant association between hazardous social habits, increasing age, overweight, and male gender and they are more prone to developing ADRs. Most of these predictors of ADRs, such as social habits, polypharmacy, and gender, are consistent with other study findings. ${ }^{[3,14]}$ The predictors such as alcoholics, smokers, and increasing age have a direct correlation with metabolism in the liver, and hence can affect the fate of drugs leading to the development of ADRs.

\section{Limitations}

The present study was carried out for a short period, and the patients included in the study were minimal. Hence, the probability of missing out the rare ADRs was more. The duration, severity, and nature of illness which could have possibly influenced the treatment decision were largely ignored. The ADRs which had the outcome of recovering were lost to follow-up because of discharge.

South Asian Journal of Cancer Volume 8 • Issue 2 • April-June 2019 


\section{Future directions}

The future studies could be conducted for a longer period so that more population can be included in the study. This study may act as a step for strengthening the pharmacovigilance activities. Further studies on the pharmacoeconomic burden on the population can be done. The patient's quality of life and quality-adjusted life years among the population with and without ADRs can be carried out.

\section{Conclusion}

Detection and prediction of ADRs in the elderly are based on monitoring and the regular review of prescribed medication. The study suggests the need for the active role of the pharmacist in studying the prescribing pattern and understanding the predictors to improve the treatment outcomes. By identifying the predictors, we can improve the prescribing pattern to decrease the pharmacoeconomic burden on the patients and also enhance the quality of life of the patients.

\section{Financial support and sponsorship}

Nil.

\section{Conflicts of interest}

There are no conflicts of interest.

\section{References}

1. Mohile S, Dale W, Hurria A. Geriatric oncology research to improve clinical care. Nat Rev Clin Oncol 2012;9:571-8.

2. Ferlay J, Shin HR, Bray F, Forman D, Mathers C, Parkin DM, et al. Estimates of worldwide burden of cancer in 2008: GLOBOCAN 2008. Int J Cancer 2010; 127:2893-917.

3. Lavan AH, Gallagher P. Predicting risk of adverse drug reactions in older adults. Ther Adv Drug Saf 2016;7:11-22

4. Korc-Grodzicki B, Boparai MK, Lichtman SM. Prescribing for older patients with cancer. Clin Adv Hematol Oncol 2014; 12:309-18.

5. Bowie MW, Slattum PW. Pharmacodynamics in older adults: A review. Am J Geriatr Pharmacother 2007;5:263-303.

6. Silva TJ, Jerussalmy CS, Farfel JM, Curiati JA, Jacob-Filho W. Predictors of in-hospital mortality among older patients. Clinics (Sao Paulo) 2009;64:613-8.

7. Sarkar A, Shahi U. Assessment of cancer care in Indian elderly cancer patients: A single center study. South Asian J Cancer 2013;2:202-8.

8. Alomar MJ. Factors affecting the development of adverse drug reactions (Review article). Saudi Pharm J 2014;22:83-94.

9. Lichtman SM, Boparai MK. Anticancer drug therapy in the older cancer patient: Pharmacology and polypharmacy. Curr Treat Options Oncol 2008;9: 191-203.

10. Maggiore RJ, Gross CP, Togawa K, Tew WP, Mohile SG, Owusu C, et al. Use of complementary medications among older adults with cancer. Cancer 2012;118:4815-23.

11. Stevenson JM, Williams JL, Burnham TG, Prevost AT, Schiff R, Erskine SD, et al. Predicting adverse drug reactions in older adults; a systematic review of the risk prediction models. Clin Interv Aging 2014;9: 1581-93.

12. Angamo MT, Curtain CM, Chalmers L, Yilma D, Bereznicki L. Predictors of adverse drug reaction-related hospitalisation in southwest ethiopia: A prospective cross-sectional study. PLoS One 2017; 12:e0186631.

13. Sharma A, Kumari KM, Manohar HD, Bairy KL, Thomas J. Pattern of adverse drug reactions due to cancer chemotherapy in a tertiary care hospital in South India. Perspect Clin Res 2015;6:109-15.

14. Shah R, Gajjar B, Desai S. A profile of adverse drug reactions with risk factors among geriatric patients in a tertiary care teaching rural hospital in India. Nat J Physiol Pharm Pharmacol 2012;2:113-22.

15. Chopra D, Rehan HS, Sharma V, Mishra R. Chemotherapy-induced adverse drug reactions in oncology patients: A prospective observational survey. Indian J Med Paediatr Oncol 2016;37:42-6.

16. Saini VK, Sewal RK, Ahmad Y, Medhi B. Prospective observational study of adverse drug reactions of anticancer drugs used in cancer treatment in a tertiary care hospital. Indian J Pharm Sci 2015;77:687-93. 